\title{
Cap-Assisted Technique versus Conventional Methods for Esophageal Food Bolus Extraction: A Comparative Study
}

\author{
Mahmoud Wahba', Ghada Habib ${ }^{2}$, Ahmed El Mazny ${ }^{1}$, May Fawzi ${ }^{1}$ Mohamed A. Elfeki ${ }^{3}$ Seham Sabry ${ }^{4}$, Mahommad ELbaz ${ }^{1}$ \\ and Sayed M Seif El Nasr ${ }^{1}$
}

${ }^{1}$ Division of Gastroenterology, Department of Internal Medicine, Cairo University, Kasr Alainy Faculty of Medicine, Cairo, ${ }^{2}$ Cairo University, Kasr Alainy Faculty of Medicine, Tropical Medicine, Cairo, ${ }^{3}$ Department of Internal Medicine, Beni-Suef University, Faculty of Medicine, Benisuef, ${ }^{4}$ Department of Internal Medicine, Al-Azhar University, Faculty of Medicine, Cairo, Egypt

Background/Aims: Food bolus impaction is the most common form of esophageal foreign body impaction observed in adults. Clinical guidelines recommend using the push technique or retrieval methods in such cases. The push technique can cause injuries in certain clinical situations. Notably, conventional retrieval methods are time and effort consuming. Cap-assisted endoscopic extraction of an impacted food bolus is an easy and effective technique; however, more data are needed for its validation. This study compared the capassisted extraction technique with conventional methods.

Methods: This prospective observational multicenter study compared the success and en bloc removal rates, total procedure time, and adverse events in both techniques..

Results: The study included 303 patients who underwent food bolus extraction. The push technique was used in 87 patients (28.7\%) and a retrieval procedure in 216 patients (71.3\%). Cap-assisted extraction was performed in 106 patients and retrieval using conventional methods in 110 patients. The cap-assisted technique was associated with a higher rate of en bloc removal $(80.2 \%$ vs. $15 \%, p<0.01)$, shorter procedure time $(6.9 \pm 3.5 \mathrm{~min}$ vs. $15.7 \pm 4.1 \mathrm{~min}, p<0.001)$, and fewer adverse events $(0 / 106 \mathrm{vs.} 9 / 110, p<0.001)$.

Conclusions: Cap-assisted extraction showed no adverse events, higher efficacy, and a shorter procedure time compared with conventional retrieval procedures. Clin Endosc 2019;52:458-463

Key Words: Foreign body; Food bolus; Cap-assisted extraction

\section{INTRODUCTION}

Foreign body ingestion is a common endoscopic emergency in both the adult and pediatric population. ${ }^{1}$ Fortunately, in most cases (80\%-90\%), the foreign body passes without intervention. However, endoscopic removal is needed in 10\%-20\% of patients, and surgery is necessary in $<1 \%$ of patients for

Received: February 5, 2019 Revised: March 18, 2019

Accepted: April 10, 2019

Correspondence: Mahmoud Wahba

Division of Gastroenterology, Department of Internal Medicine, Faculty of Medicine, Cairo University, 130 El-Hay Eltalt, Mokattam, Cairo, Egypt

Tel: +20-1-123326663, Fax: +20-2-23682030, E-mail: Mahmoud.wahba@kasralainy.edu.eg

ORCID: https://orcid.org/0000-0001-5263-9103

(c) This is an Open Access article distributed under the terms of the Creative Commons Attribution Non-Commercial License (http://creativecommons.org/ licenses/by-nc/3.0) which permits unrestricted non-commercial use, distribution, and reproduction in any medium, provided the original work is properly cited. foreign body extraction or for the management of complications. $^{2-8}$ Various types of foreign body such as blunt or sharp objects are encountered in clinical practice in addition to food bolus (FB) impaction. The latter is more likely to occur in adults than in children and more commonly occurs in the elderly population and in those with underlying esophageal pathology. ${ }^{9}$ Patients usually present with sore throat, dysphagia, odynophagia, and retrosternal pain or discomfort. Although patients can usually localize the site of discomfort, it may not accurately correlate with the site of FB impaction. ${ }^{3,4,6}$

FB obstruction requires urgent endoscopic removal within 24 hours to avoid complications (e.g., perforation, retropharyngeal abscess, or fistula formation) and within 6 hours in patients with manifestations of complete esophageal obstruction (e.g., the inability to swallow liquids or hypersalivation) because these patients are at a high risk of aspiration and/or pressure necrosis. ${ }^{9}$ 
The European Society of Gastrointestinal Endoscopy (ESGE) recommends the push technique as the primary method to treat FB obstruction. ${ }^{10}$ Careful assessment of any distal obstructive esophageal pathology is performed followed by gentle pushing of the impacted FB towards the stomach. In cases where significant resistance is encountered, en bloc retrieval of the FB or piecemeal removal after fragmentation should be attempted using any of the available endoscopic accessories such as grasping forceps, polypectomy snares, retrieval net, or the Dormia basket. ${ }^{2,311,12}$ Notably, the push technique is not always safe or feasible in clinical practice and may cause serious adverse effects (e.g., esophageal perforation), particularly in patients with underlying esophageal pathology. Moreover, retrieval of the FB using any of the aforementioned methods may be associated with fragmentation of the bolus, which makes the procedure more difficult and time consuming (Fig. 1).

En bloc removal using cap-assisted endoscopic extraction is an easy and rapid technique during which a transparent cap is applied to the tip of the gastroscope for retrieval of the FB using continuous suction. Two recent randomized studies demonstrated the efficacy of the cap-assisted technique in the extraction of a bony upper esophageal $\mathrm{FB},{ }^{13}$ as well as in relieving boneless FB obstruction. ${ }^{14}$

Our study compared the cap-assisted technique with conventional methods for the removal of FB obstruction.

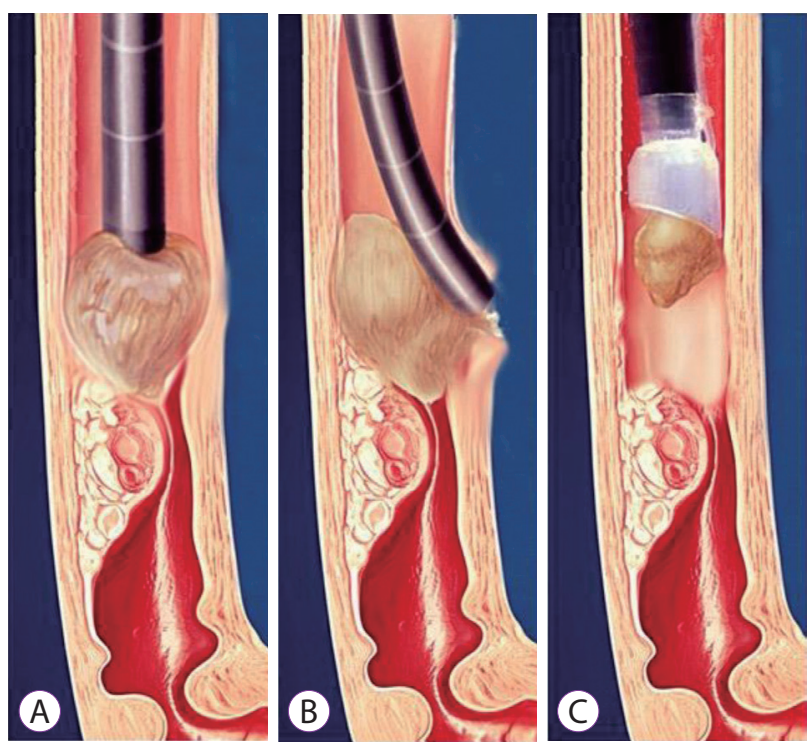

Fig. 1. Image showing food bolus impaction at the distal esophagus. (A) Underlying lesion can be observed preventing the use of the push technique. (B) Esophageal perforation is likely if the pushing action is continued blindly. (C) Cap-assisted extraction serves as an easy method of food bolus extraction.

\section{PATIENTS AND METHODS}

This prospective observational multicenter study was performed across the following tertiary referral hospitals: The Internal Medicine Department, Cairo University Hospital, AlAzhar University Hospital, and Beni-Suef University Hospital. All patients underwent emergency endoscopy for FB obstruction between May 2016 and November 2018. All patients provided written informed consent. The following outcomes were measured: the success rate of $\mathrm{FB}$ extraction, procedure time, rate of en bloc FB removal, and procedure-related adverse events. The study protocol conforms to the ethical guidelines of the Declaration of Helsinki.

\section{Procedure}

All endoscopies were performed by senior gastroenterologists well-experienced in the management of FB obstruction, using GIF 140, 160, 170, 180 Olympus endoscopes (Olympus Optical Co., Tokyo, Japan). Gastroscopies were performed under topical pharyngeal anesthesia using lidocaine spray. The choice of endoscopic technique and devices used to remove FB were at the endoscopist's discretion on a case-by-case basis. (1) The push technique was performed as follows: the FB was gently pushed towards the stomach. If significant resistance was encountered, the procedure was switched to a retrieval method for FB extraction. (2) Conventional methods were used as follows: extraction of the FB obstruction was performed using one or more retrieval devices including a polypectomy snare, a net retriever, grasping forceps, and a Dormia basket. (3) The cap-assisted technique was performed as follows: a transparent band ligation cap was used (the six-shooter multi-band kit, MBL-6; Cook Inc., Winston-Salem, NC, USA). The cap was fixed to the tip of the endoscope, and constant suction was applied to fully engage the FB into the cap. The scope was subsequently carefully withdrawn along with the dislodged FB (Fig. 2).

If retrieval of the FB was not possible using the initial technique, the procedure was switched to another retrieval method or combined procedures were attempted. After removal of the FB, we carefully assessed any underlying esophageal pathology. Additionally, endoscopic biopsy specimens were obtained from abnormal-appearing mucosa, and we also evaluated procedure-related adverse events such as mucosal tears, bleeding, or perforation.

\section{Data collection}

The following data were obtained: age, sex, presenting symptoms, history of esophageal disease, procedure time (time needed for the endoscopist to successfully remove the impacted FB [recorded from the time of esophageal evaluation at the 
start of the esophagogastroduodenoscopy to complete removal of the FB]), success rate of each technique, rate of en bloc removal of the FB (defined as the rate of complete removal of the FB without esophageal food residue), and the incidence of procedure-related adverse events (mucosal tears, bleeding, or perforation).

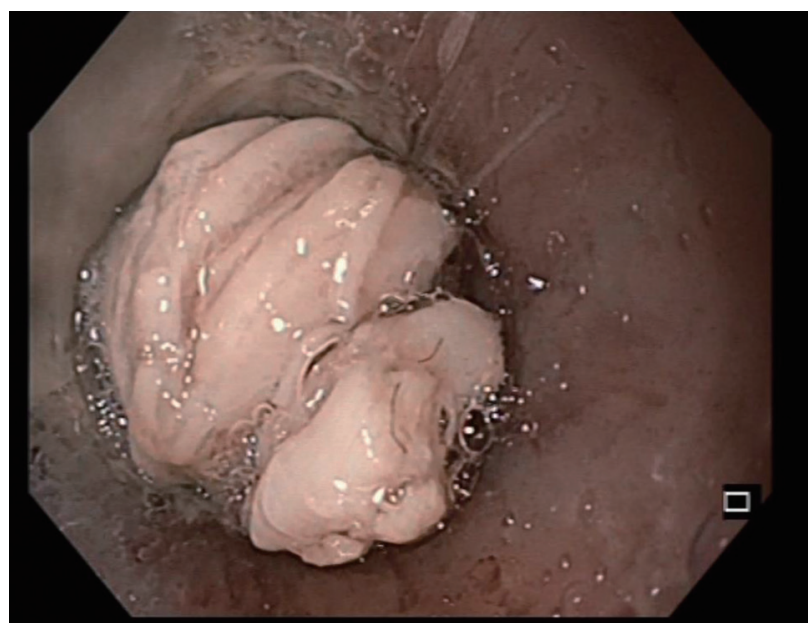

Fig. 2. Image showing the technique of cap-assisted extraction. The cap is applied with a slight twisting movement, and continuous suction is performed with gentle withdrawal of the scope.

\section{Statistical analysis}

Descriptive statistics were used for data analysis. Numerical data were expressed as mean (standard deviation) and categorical data as frequencies and percentages. Continuous variables were tested for normality using the Shapiro-Wilk normality test. An intergroup comparison was performed, and normally distributed data were analyzed using the independent samples $t$-test, and non-normally distributed data were analyzed using the Mann-Whitney U test. The Chi-squared test or the Fischer exact test was used to compare percentages between different groups of patients. Data analysis was performed using the STATA software, version 13.1.

\section{RESULTS}

A total of 303 patients underwent gastroscopy for FB obstruction. In 87 patients (28.7\%), the FB could be gently pushed towards the stomach. In 216 patients $(71.3 \%)$, a retrieval method was required for FB removal. In 106 patients (35.0\%), the cap-assisted technique was used, and in 110 patients (36.3\%) conventional methods were used for FB extraction.

Demographic and clinical characteristics of patients The mean age of the study population was 47.4 years (19-78

Table 1. Clinical Characteristics of Patients in Both Groups

\begin{tabular}{|c|c|c|c|}
\hline & $\begin{array}{c}\text { Cap-assisted group } \\
n=106\end{array}$ & $\begin{array}{c}\text { Conventional group } \\
n=110\end{array}$ & $p$-value \\
\hline Mean age (yr) & 51.7 & 52.9 & $>0.5$ \\
\hline Male:Female ratio & $0.71(44: 62)$ & $0.77(48: 62)$ & $>0.5$ \\
\hline Presenting symptom & & & $>0.5$ \\
\hline FB sensation & $106(100 \%)$ & $110(100 \%)$ & \\
\hline Odynophagia & $106(100 \%)$ & $110(100 \%)$ & \\
\hline Dyspahgia & $106(100 \%)$ & $110(100 \%)$ & \\
\hline Chest pain & $30(28.3 \%)$ & $29(26.4 \%)$ & \\
\hline Hypersalivation & $10(9.4 \%)$ & $12(12.9 \%)$ & \\
\hline Underling esophageal pathology & & & $>0.5$ \\
\hline None & $37(34.9 \%)$ & $34(30.9 \%)$ & \\
\hline Post-corrosive esophagitis & $32(30.2 \%)$ & $30(27.3 \%)$ & \\
\hline Peptic stricture & $21(19.8 \%)$ & $24(21.8 \%)$ & \\
\hline Schatzki ring & $5(4.71 \%)$ & $5(4.71 \%)$ & \\
\hline Eosinophilic esophagitis & $4(3.8 \%)$ & $6(5.5 \%)$ & \\
\hline Esophageal tumor & $4(3.8 \%)$ & $6(5.5 \%)$ & \\
\hline Esophagitis & $2(1.9 \%)$ & $1(0.9 \%)$ & \\
\hline Anastomotic stricture & $1(0.9 \%)$ & $4(3.6 \%)$ & \\
\hline
\end{tabular}

FB, food bolus. 
years), and the study included 149 men and 154 women. No significant difference was observed between patients in the conventional and the cap-assisted groups in age, sex, or presenting symptoms (Table 1). The main presenting symptoms were foreign body sensation, followed by odynophagia, and dysphagia.

Underlying esophageal pathology could be identified in 201 patients (66.3\%), and post corrosive esophagitis was the most common condition observed. No intergroup difference was observed in the types of underlying esophageal pathology. No significant intergroup difference was observed in the site of FB impaction in that most FBs were identified in the upper esophagus. A polypectomy snare was the most common endoscopic accessory device used for FB retrieval (Table 2).

\section{Procedure time, efficacy and complications}

The cap-assisted technique required a significantly shorter procedure time than conventional retrieval methods $(6.9 \pm 3.5$ min vs. $15.7 \pm 4.1 \mathrm{~min}, p<0.001)$ and showed a higher rate of $e n$ bloc removal ( $80.1 \%$ vs. $15.0 \%, p<0.01$ ) (Figs. 3,4 ).

Notably, in 15 patients (5.0\%) in whom retrieval using conventional methods failed, the procedure was switched to a cap-assisted technique. In 7 patients (2.3\%), the cap-assisted technique was not feasible because the scope with the cap could not be advanced to the site of FB impaction secondary to a long narrowed esophagus before the FB. In these patients, FB retrieval was achieved using a conventional method.

The cap-assisted technique was associated with significantly fewer adverse events (0/106 vs. 9/110, $p=0.001)$.

Mild local adverse events occurred in patients in whom the conventional technique was used -5 patients developed superficial mucosal tears, and 4 patients developed mild regional bleeding that did not require blood transfusion. No procedure-related mortality occurred with use of either tech-

Table 2. Site of Food Bolus Obstruction and Accessory Device Used in Both Groups

\begin{tabular}{lcc}
\hline & $\begin{array}{c}\text { Cap-assisted group } \\
\boldsymbol{n}=\mathbf{1 0 6}\end{array}$ & $\begin{array}{c}\text { Conventional group } \\
\boldsymbol{n}=\mathbf{1 1 0}\end{array}$ \\
\hline Site of FB impaction & & \\
Upper & $46(43.4 \%)$ & $49(44.6 \%)$ \\
Middle & $37(34.9 \%)$ & $34(30.9 \%)$ \\
Lower & $23(21.7 \%)$ & $27(24.5 \%)$ \\
Endoscopic device used for FB removal, $n(\%)$ & & $91(82.7 \%)$ \\
Polypectomy snare & 0 & $78(70.9 \%)$ \\
Rat-tooth forceps & 0 & 0 \\
Cap & $106(100 \%)$ & \\
\hline
\end{tabular}

$\mathrm{FB}$, food bolus.

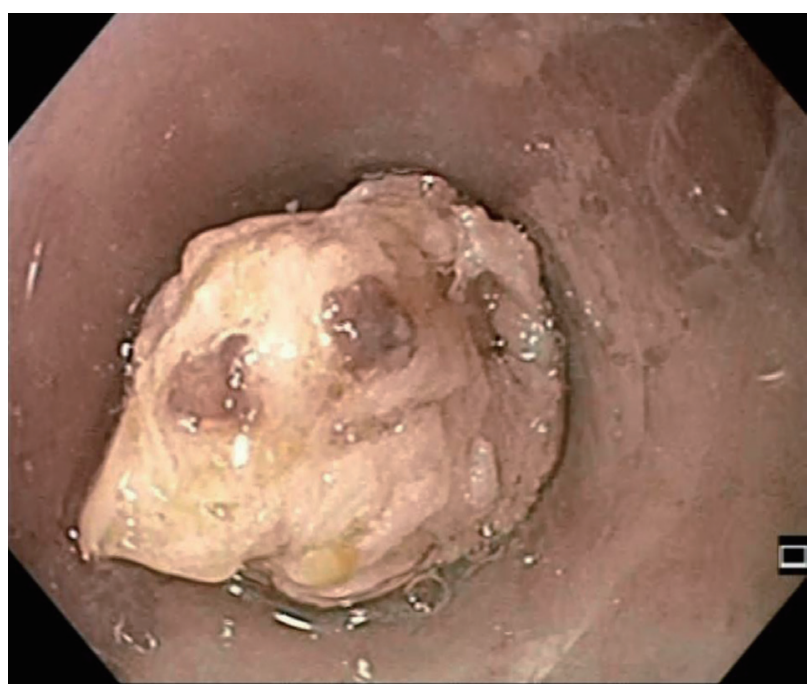

Fig. 3. Image showing the food bolus is captured within the cap during extraction.

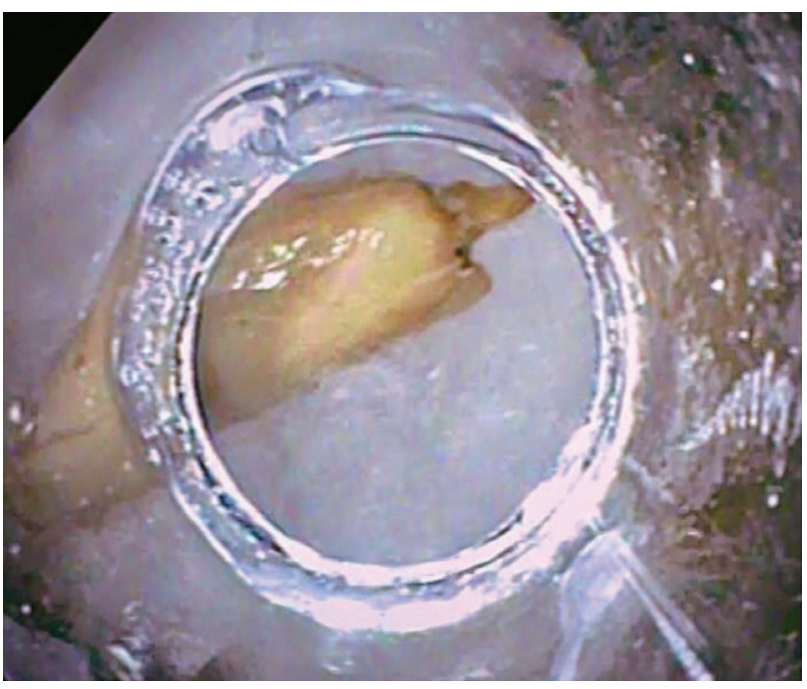

Fig. 4. Image showing cap-assisted en bloc food bolus extraction. 
nique (Table 3). Patients who developed procedure-related adverse events required admission for a few days for medical treatment and clinical observation and were subsequently discharged without long-term complications.

\section{DISCUSSION}

Esophageal FB obstruction is commonly encountered in clinical practice, particularly in adults with underlying esophageal pathology. The ESGE recommends a trial of a gentle push technique as the first-line treatment to attempt pushing the FB towards the stomach. ${ }^{10}$ However, this method may cause serious adverse events, particularly in patients with underlying esophageal pathology. If marked resistance is encountered while pushing the FB, retrieval of the FB should be attempted. En bloc removal of FB obstruction using conventional retrieval methods is usually difficult because the FB undergoes fragmentation, making extraction time and effort consuming.

The endoscopy cap often bridges the 'can to could'. Thus, it is among the best inventions in the domain of endoscopy. The cap adds practical functionality to the endoscope in that some of the functions performed by the endoscope cannot be accomplished without the cap. A transparent cap allows better visualization and improves the suction capacity by enlarging the area of suction to the opening of the cap rather than the opening of the channel. ${ }^{15-18}$

A previous prospective study has validated the efficacy of the cap-assisted technique in boneless FB obstruction extraction confirming that it required a shorter procedure time than conventional methods ( $34.3 \pm 8.0 \mathrm{~min}$ vs. $43.3 \pm 22.6 \mathrm{~min}$ ), achieved a higher rate of en bloc removal of FB obstruction (87.3\% vs. $22.8 \%)$, and showed fewer adverse events. ${ }^{14}$ In our study, we prospectively included all patients undergoing upper endoscopy for boneless FB obstruction between 2016 and 2018, and conventional as well as cap-assisted techniques were used for FB extraction. The cap-assisted technique achieved a higher rate of en bloc FB removal (80.1\% vs. $15.0 \%$, $p<0.001)$ and showed a shorter procedure time ( $6.9 \pm 3.5 \mathrm{~min}$ vs. $15.7 \pm 4.1$ min, $p<0.001)$ than conventional methods, and these results concur with the study reported by Ooi et al. ${ }^{14}$ Application of the cap and continuous suction successfully engage the FB inside the cap with its longest axis parallel to the scope, which allows complete removal as a single easy step with less contact with the mucosal surface, thereby protecting the mucosa from the risk of manipulation-induced injury. The procedure time observed in our study was shorter than that reported by Ooi et al. ${ }^{14}$ However, we only considered the time needed for esophageal assessment and removal of FB obstruction without considering the time needed for assembly of the endoscope and administration of anesthesia. No procedure-related mortality and no adverse events were observed in the cap-assisted group compared with the few adverse events observed in the conventional methods group (0/106 vs. 9/110, $p<0.001)$.

Most patients showed underlying esophageal pathology (163/253), as was reported by previous studies. ${ }^{13,14}$ Most patients presented with post-corrosive esophagitis; therefore, FB obstruction was most commonly detected in the upper esophagus. The upper esophagus is one of the narrowest areas of the gastrointestinal tract, and manipulation in this area is usually difficult with a high risk of aspiration. The application of the cap allows better visualization of the operative field because it opens the physiological space, and the procedure can be performed easily and rapidly. Nevertheless, the cap-assisted technique is not always feasible-in $2.3 \%$ of patients, the scope with the applied cap could not be advanced to the site of FB obstruction owing to marked stenosis of the upper esophagus.

Our study is a multicenter study that was performed over 2 years and included an adequate number of patients with FB obstruction. The number of patients included in this study was greater than that in previous studies. The study performed a prospective analysis of patients. In our study, randomization was not feasible because the initial endoscopic assessment significantly affected the choice of method selected for FB obstruction extraction owing to proximal strictures that prevented the passage of the cap applied to the tip of the endoscope. Moreover, in patients with suspected malignancy

Table 3. Procedure Time, Success Rate and Procedure-Related Adverse Events

\begin{tabular}{|c|c|c|c|}
\hline & $\begin{array}{c}\text { Cap-assisted group } \\
n=106\end{array}$ & $\begin{array}{l}\text { Conventional group } \\
\qquad n=110\end{array}$ & $p$-value \\
\hline Procedure time (min) & $6.9 \pm 3.5$ & $15.7 \pm 4.1$ & $<0.001$ \\
\hline Rate of en bloc removal & $80.2 \%$ & $15.5 \%$ & $<0.001$ \\
\hline Procedure-related adverse events, $n(\%)$ & & & $<0.001$ \\
\hline Superficial mucosal injury & 0 & $5(4.5 \%)$ & \\
\hline Regional bleeding & 0 & $4(3.6 \%)$ & \\
\hline
\end{tabular}


or in those with ulcerated esophageal mucosa, we did not use the push technique to avoid complications. Thus, the method of FB obstruction extraction was tailored on the basis of the initial endoscopic assessment. Moreover, data regarding the exact size of the FB could not be obtained in most patients because in some cases, the FB was pushed towards the stomach and in others, the FB was removed in a piecemeal manner. Theoretically, the size of the FB might have affected the procedure time or the success rate.

In conclusion, cap-assisted removal of an impacted esophageal FB is associated with a higher en bloc extraction rate, shorter procedure time, and fewer adverse events. This procedure is easy to perform and does not require specialized training or long-term experience compared with conventional methods. However, it may not always be feasible. We propose that this technique should be considered as a useful option for removal of an impacted esophageal FB.

\section{Conflicts of Interest}

The authors have no financial conflicts of interest.

\section{REFERENCE}

1. Longstreth GF, Longstreth KJ, Yao JF. Esophageal food impaction: epidemiology and therapy. A retrospective, observational study. Gastrointest Endosc 2001;53:193-198.

2. Ambe P, Weber SA, Schauer M, Knoefel WT. Swallowed foreign bodies in adults. Dtsch Arztebl Int 2012;109:869-875.

3. ASGE Standards of Practice Committee, Ikenberry SO, Jue TL, et al. Management of ingested foreign bodies and food impactions. Gastrointest Endosc 2011;73:1085-1091.

4. Dray X, Cattan P. Foreign bodies and caustic lesions. Best Pract Res Clin Gastroenterol 2013;27:679-689.
5. Ko HH, Enns R. Review of food bolus management. Can J Gastroenterol 2008;22:805-808.

6. Pfau PR. Removal and management of esophageal foreign bodies. Tech Gastrointest Endosc 2014;16:32-39.

7. Sugawa C, Ono H, Taleb M, Lucas CE. Endoscopic management of foreign bodies in the upper gastrointestinal tract: a review. World J Gastrointest Endosc 2014;6:475-481.

8. Telford JJ. Management of ingested foreign bodies. Can J Gastroenterol 2005;19:599-601.

9. Magalhães-Costa P, Carvalho L, Rodrigues JP, et al. Endoscopic management of foreign bodies in the upper gastrointestinal tract: an evidence-based review article. GE Port J Gastroenterol 2016;23:142-152.

10. Birk M, Bauerfeind P, Deprez PH, et al. Removal of foreign bodies in the upper gastrointestinal tract in adults: European Society of Gastrointestinal Endoscopy (ESGE) clinical guideline. Endoscopy 2016;48:489496.

11. Ciriza C, García L, Suárez P, et al. What predictive parameters best indicate the need for emergent gastrointestinal endoscopy after foreign body ingestion? J Clin Gastroenterol 2000;31:23-28.

12. Vicari JJ, Johanson JF, Frakes JT. Outcomes of acute esophageal food impaction: success of the push technique. Gastrointest Endosc 2001;53:178181.

13. Zhang S, Wang J, Wang J, Zhong B, Chen M, Cui Y. Transparent cap-assisted endoscopic management of foreign bodies in the upper esophagus: a randomized, controlled trial. J Gastroenterol Hepatol 2013;28:1339-1342.

14. Ooi M, Young EJ, Nguyen NQ. Effectiveness of a cap-assisted device in the endoscopic removal of food bolus obstruction from the esophagus. Gastrointest Endosc 2018;87:1198-1203.

15. Zhang S, Cui Y, Gong X, Gu F, Chen M, Zhong B. Endoscopic management of foreign bodies in the upper gastrointestinal tract in South China: a retrospective study of 561 cases. Dig Dis Sci 2010;55:1305-1312.

16. Lee JS, Chun HJ, Lee JM, et al. Salvage technique for endoscopic removal of a sharp fish bone impacted in the esophagus using a transparent cap and detachable snares. Korean J Gastroenterol 2013;61:215-218.

17. Inoue $\mathrm{H}$, Endo M, Takeshita K, Yoshino K, Muraoka Y, Yoneshima H. A new simplified technique of endoscopic esophageal mucosal resection using a cap-fitted panendoscope (EMRC). Surg Endosc 1992;6:264-265.

18. Soehendra N, Seewald S, Groth S, et al. Use of modified multiband ligator facilitates circumferential EMR in Barrett's esophagus (with video). Gastrointest Endosc 2006;63:847-852. 